\title{
The application of the photoacoustic transmittance oscillations for determining elastic constants in gallium and indium selenides
}

\author{
Ch. Ferrer, A. Segura, M. V. Andrés, V. Muñoz, and J. Pellicer \\ Departamento de Física Aplicada, Universidad de Valencia, Dr. Moliner 50, 46100 Burjassot, Valencia, \\ Spain
}

(Received 9 August 1995; accepted for publication 28 November 1995)

Transmittance periodic oscillations are observed in GaSe and InSe on excitation with optical pulses. Such oscillations are explained in terms of photoacoustic generation of dilatational waves, which become resonant within the crystal. Spectral analysis of those oscillations in samples of different thickness has led to an accurate determination of the longitudinal acoustic-wave velocity along the crystallographic axis c. (C) 1996 American Institute of Physics. [S0021-8979(96)07005-5]

\section{INTRODUCTION}

The photoacoustic effect has been widely used in the past years. In the field of vibrating sensors, the mechanical resonance can be excited by means of light pulses. ${ }^{1}$ The photoacoustic spectroscopy has been a useful tool in determining the local thermal properties of samples, with important applications in semiconductors devices. ${ }^{2}$ Photothermal deflection spectroscopy ${ }^{3}$ has allowed the observation of the transient expansion of heated surfaces and has subsequently been used to observe the surface motion of thin films, ${ }^{4}$ whereas supplementary mechanism for photoacoustic generation in semiconductors (mechanical strain induced through fluctuations in the photogenerated free-carrier density) $)^{5,6}$ has been observed with this beam deflection technique.

The pump and probe technique has been previously used in semiconductors characterization to study the generation and detection mechanism of coherent acoustic phonons in thin films. ${ }^{8-10}$ Thomsen et al. proposed such a technique for the measurement of velocity and attenuation of highfrequency phonons. There, the same source (1 ps pulses) was used for the production and detection of photoinduced transmission and reflection oscillations in thin films $(0.05-0.12$ $\mu \mathrm{m})$ of $a-\mathrm{As}_{2} \mathrm{Te}_{3}$ and cis-polyacetylene. The same technique was applied by Wiensenfield to InGaAsP films $(0.2-0.6$ $\mu \mathrm{m})$. Both assumed that the pump pulse induces a thermal strain which is the only responsible for the transmission oscillations and hence for a variation of the absorption coefficient of the sample.

In this study, we use the pump ( $\sim 10 \mathrm{~ns})$ and probe technique with different sources to study photoinduced oscillations in the transmittance of GaSe and InSe samples (10-100 $\mu \mathrm{m})$. Such oscillations have been observed to occur at welldefined frequencies, to have a long decay time $(\sim 10 \mu \mathrm{s})$ and to allow for an accurate determination of the longitudinal acoustic-wave velocity parallel to the $c$ axis. In our case it is the refractive index which is mainly affected by the longitudinal waves which become resonant within the sample, and an estimate of the optical path variation caused by the light pulses is done, based on measurements of the oscillations amplitude at different temperatures.

The layer-type semiconductors GaSe and InSe are hexagonal crystals with a strong anisotropy. The crystals were grown by the Bridgman-Stockbarger method from the ma- terial previously synthesized from direct combination of the elements. Thin slabs with good mirrorlike surfaces can be easily cleaved in a plane normal to the $c$ axis of the crystal, using a simple razor blade. GaSe is a direct semiconductor with an energy gap of about $2.0 \mathrm{eV}$ at room temperature, while InSe is an indirect semiconductor with an energy gap of about $1.2 \mathrm{eV}$.

\section{EXPERIMENTAL SETUP}

Figure 1 shows the experimental arrangement. A NdYAG laser, combined with either a dye laser or a Ti-sapphire laser, works as a pump laser, providing optical pulses of 7-8 ns width (half-maximum amplitude), $10 \mathrm{~Hz}$ repetition rate, and a maximum energy of $3 \mathrm{~mJ}$. A wavelength of $563 \mathrm{~nm}$ from the dye laser $(h \nu \approx 2.2 \mathrm{eV})$ has been used for the GaSe samples and a wavelength of $740 \mathrm{~nm}$ from the Ti-sapphire laser $(h \nu \approx 1.7 \mathrm{eV})$ for the InSe samples. A lens of $50 \mathrm{~cm}$ focal length and a beam splitter are used in order to focus the beam and reduce its intensity, respectively, giving a spot of 3 $\mathrm{mm}$ on the sample. The probe laser beam, set parallel to the $c$ axis and polarized perpendicularly to it, is used for inspection of the transmittance. A visible He-Ne laser $(633 \mathrm{~nm}, 5$ $\mathrm{mW}$ ) has been used in the case of GaSe samples and an infrared He-Ne laser $(1550 \mathrm{~nm}, 1 \mathrm{~mW})$ in the case of InSe samples. The samples are mounted on an aluminium gasket, with a hole of $2 \mathrm{~mm}$ diameter, and then on a sample holder which includes a heating element and a thermistor for temperature measurements. The transmitted beam is detected by a photoreceiver of about $10^{4} \mathrm{~V} / \mathrm{W}$ gain and a DC- $125 \mathrm{MHz}$ nominal frequency band, based on either a Si photodiode or an InGaAs photodiode. The photoreceiver is positioned at the exit of a monochromator which allows for the selection of the probe beam wavelength. The detector output signal is subsequently acquired, using the laser synchronism output as a trigger.

The sample thickness has been accurately measured by a fitting procedure which takes into account the position of all interference maxima in a large spectral range, and the wavelength dispersion of the refractive index. The set of cleaved samples have the following thickness: 8.48, 12.8, 15.04, $20.0,23.01,27.25,32.59,45.65$, and $95.61 \mu \mathrm{m}$ in the case of GaSe, and 29.02, 39.59, 19.97, 55.76, and 59.51 $\mu \mathrm{m}$ in the case of InSe. 


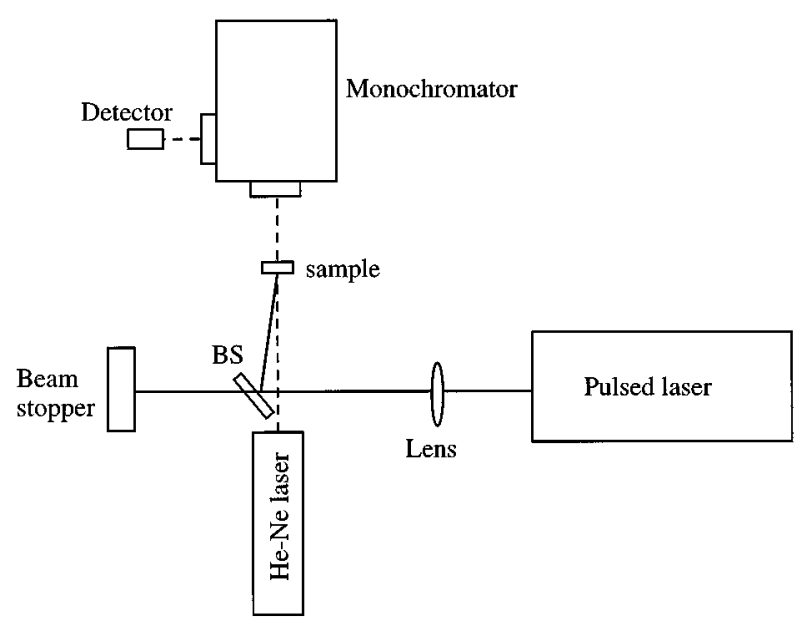

FIG. 1. Experimental arrangement.

\section{MEASUREMENTS AND DATA ANALYSIS}

Figure 2(a) shows a typical transmittance signal obtained for a GaSe sample. After a constant value, a negative peak appears in coincidence with the pump laser pulse. Such a sudden variation has been studied elsewhere ${ }^{11}$ and is caused by a decrease in the refractive index due to the exciton screening by photogenerated carriers. Then the transmittance dc value increases as a consequence of the sample heating and some oscillations at a definite frequency are observed.
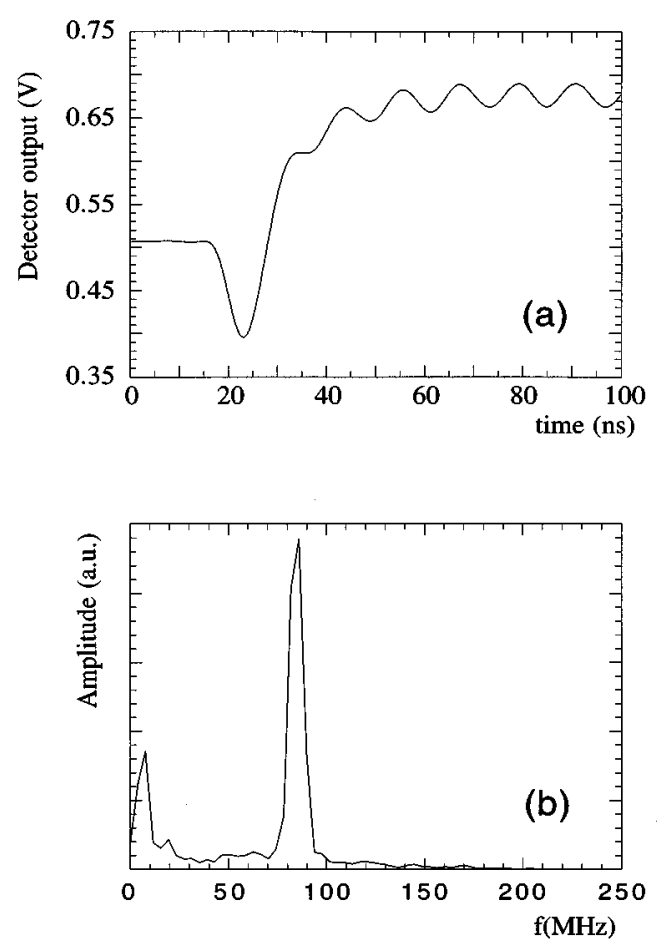

FIG. 2. (a) Signal corresponding to the probe laser intensity transmitted by a GaSe sample of $15.04 \mu \mathrm{m}$ thickness, measured in coincidence with the pump laser pulse. (b) Amplitude spectrum of the signal after high pass filtering $(5 \mathrm{MHz})$.
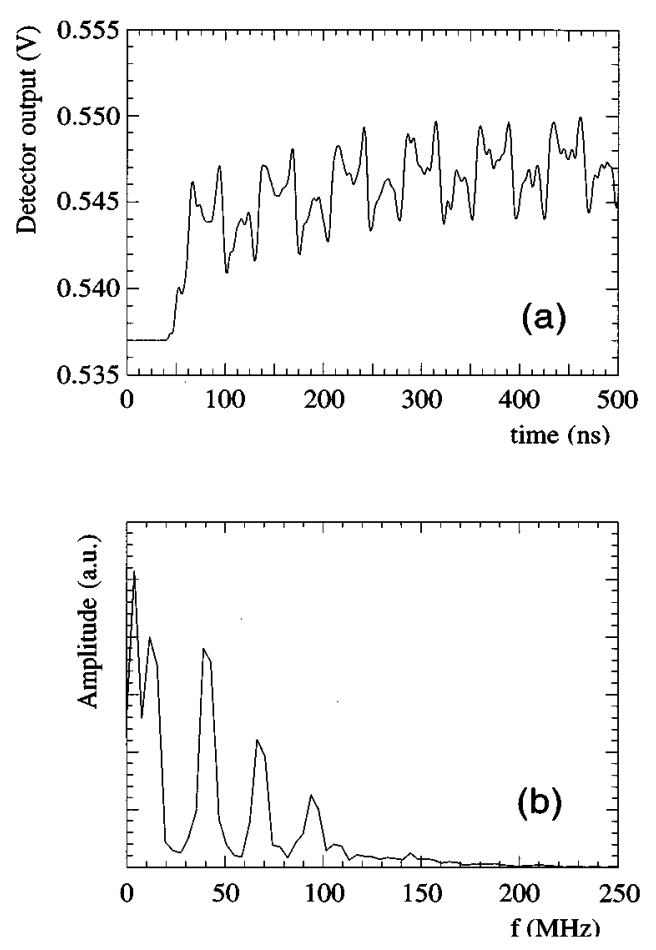

FIG. 3. (a) Signal corresponding to the probe laser intensity transmitted by a GaSe sample of $95.61 \mu \mathrm{m}$ thickness, measured in coincidence with the pump laser pulse. (b) Amplitude spectrum of the signal after high pass filtering $(5 \mathrm{MHz})$.

The period of such oscillations keep a linear relation with the sample width and in the case of the thickest samples, harmonics at higher frequencies appear [see, for example, Fig. 3(a)]. Such results suggest a photoacoustic effect: the pump laser beam impinges on the sample inducing a strain which generates acoustic waves, some of which become resonant within the sample width. Such standing longitudinal waves should, by successive compression and expansion of the sample, lead to variations in the optical path $\Delta(n d)$ and then to oscillations in transmitted probe-beam intensity. The different acoustic resonances should appear with wavelengths $\lambda_{a}=2 d /(2 m+1), \quad m=0,1,2$, etc., corresponding to the fundamental resonance and the first, second, etc., harmonics, respectively. The associated frequencies should be 1, 3, 5, etc., times the fundamental frequency $f_{0}=v / 2 d$, with $v$ the longitudinal acoustic-wave velocity along the $c$ axis.

We can provide a more general description in terms of the acoustic fields and waves in the solid. The semiconductor sample can be regarded as an unbounded plate waveguide. The acoustic mode spectrum for an isotropic plate ${ }^{12}$ can be classified into SH modes (horizontal shear waves) and Lamb modes (coupled vertical shear and pressure waves). The Lamb waves can be either symmetric or antisymmetric and are called dilatational $\left(L_{n}\right)$ and flexural $\left(F_{n}\right)$ waves, respectively. The excitation of a particular wave is determined by the matching between the volume dilatation generated by the light pulses and the volume dilatation associated with the mode. ${ }^{5}$ In our case, since the laser spot is about $3 \mathrm{~mm}$, the acoustic modes excited in the sample will exhibit a wave- 
length along the plate of the same order as the spot size. Thus, the propagation constant $\beta$ of each mode will be rather small. In such a case, when $\beta \approx 0$, both the symmetric $\left(L_{n}\right)$ and antisymmetric $\left(F_{n}\right)$ Lamb waves are either pure shear or pure longitudinal transverse standing waves. To be exact, the $L_{1}, L_{3}, L_{5}$, etc., $F_{2}, F_{4}, F_{6}$, etc., are shear type waves, and the $L_{2}, L_{4}, L_{6}$, etc., $F_{1}, F_{3}, F_{5}$, etc., are longitudinal type waves. Furthermore, while the photoacoustic effect can only excite the longitudinal type waves, our probe beam can not sense the flexural modes where no overall optical path variation is generated. To conclude, only the series $L_{2}, L_{4}, L_{6}$, etc., relates to our experiment. When $\beta=0$ such a series satisfies the condition $\lambda_{a}=2 d /(2 m+1)$, with $m=0,1,2, \ldots$. $\left(\lambda_{a}=v / f\right)$, stated in the previous paragraph.

In order to verify the picture described above, the amplitude spectra of signals acquired at room temperature have been calculated: data have been high pass filtered with a numerical sixth-order Butterworth filter, a Hanning window has been applied in order to reduce leakage and FFT of the resulting signals have been performed. Occasionally the signals have been padded with extra constant values in order to increase the frequency resolution in the spectrum. Figures 2(b) and 3(b) show the amplitude spectra corresponding to transmittance data shown in Figs. 2(a) and 3(a), respectively. Whereas in Fig. 2(b) there is just one frequency (fundamental) peak, in 3(b) higher harmonics are present: the first (highest amplitude) peak is just a filtering effect on lowfrequency background and it is the second peak, which emerges from background at $13.5 \mathrm{MHz}$, the lowest (fundamental) frequency corresponding to transmittance oscillations, and successive peaks at $41,68.4$, and $95.7 \mathrm{MHz}$ are harmonics corresponding to 3,5 , and 7 times the fundamental frequency, respectively. The thinnest sample $(8.48 \mu \mathrm{m})$ presents a very low amplitude oscillating pattern and the resonant frequency peak can hardly be identified from background. Such a behavior can be justified taking in account the detector frequency bandwidth (for the same reason harmonics are often cut out).

Linearity of the transmittance oscillations amplitude versus the pump laser energy ${ }^{5}$ has been also verified within the accuracy of our experiments.

Such results confirm our hypothesis of resonant photogenerated dilatational waves which modify the sample transmittance. Furthermore, they provide a simple and precise method to determine the longitudinal acoustic-wave velocity along $c$ axis by a least-squares fit of frequency versus $\lambda_{a}^{-1}$. Forcing the fit to pass through the origin, as it is shown in Fig. 4(a), we determine the slope $v=2595 \pm 11 \mathrm{~m} / \mathrm{s}$ for the GaSe samples. This value is consistent with the values found in the literature: a longitudinal speed of $2482 \mathrm{~m} / \mathrm{s}$ obtained by acoustic pulse techniques at $1.67 \mathrm{MHz},{ }^{13} c_{33}$ $=3.51 \times 10^{10} \mathrm{Nm}^{-2}(v=2642 \mathrm{~m} / \mathrm{s})$ obtained by Brillouin scattering ${ }^{13}$ and $2606 \mathrm{~m} / \mathrm{s}$ in a recent experiment ${ }^{14}$ (echopulses technique at $30 \mathrm{MHz}$ ). Figure 4(b) shows the corresponding linear fit for the InSe samples, which gives a longitudinal velocity parallel to $c$ axis $v=2462 \pm 15 \mathrm{~m} / \mathrm{s}$, consistent with the tabulated value $c_{33}=3.82 \times 10^{10} \mathrm{Nm}^{-2}$ $(v=2624 \mathrm{~m} / \mathrm{s})$, obtained by acoustic pulse techniques at 10 MHz. ${ }^{13}$
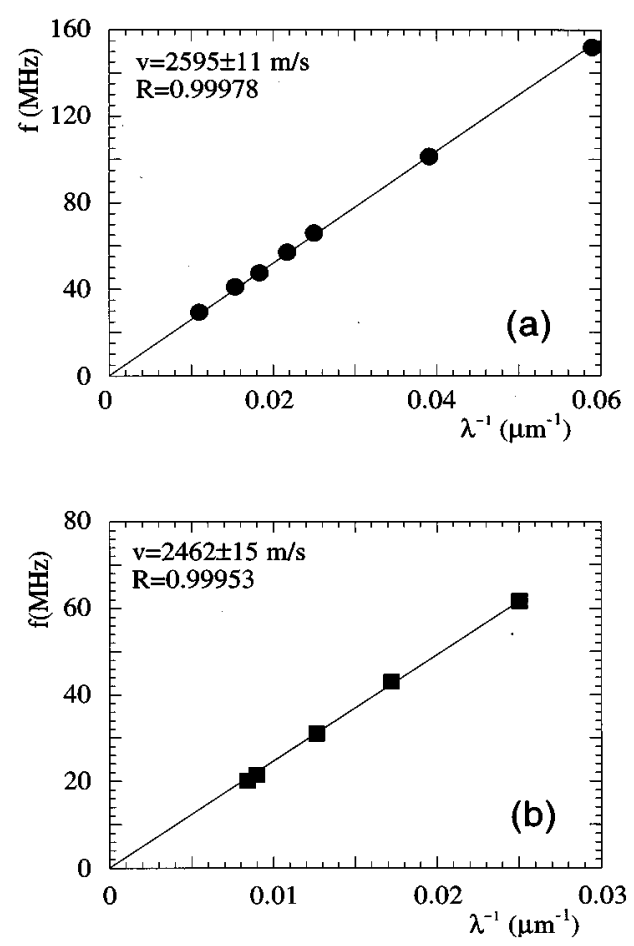

FIG. 4. Transmittance oscillations frequency vs the acoustic wave number $\lambda^{-1}$. The linear fit gives the longitudinal acoustic-wave velocity: (a) GaSe $(v=2595 \mathrm{~m} / \mathrm{s})$ and (b) InSe $(v=2462 \mathrm{~m} / \mathrm{s})$.

Further information can be obtained by measuring simultaneously the transmittance $(T)$ of the sample and the amplitude $\left(A_{\text {osc }}\right)$ of the photoacoustic induced transmittance oscillations, for different temperatures. The transmittance of the sample is that of a Fabry-Perot for a plane wave at normal incidence:

$$
T=\frac{(1-R)^{2} e^{-\alpha d}}{1+R^{2} e^{-2 \alpha d}-2 R e^{-\alpha d} \cos (4 \pi n d / \lambda)},
$$

$\alpha$ being the absorption coefficient, $n$ the refractive index, $d$ the sample thickness, and $R$ the reflection coefficient.

If we focus on GaSe samples, the temperature affects the transmittance through both $n$ and $\alpha$, whose temperature dependence is well known. ${ }^{15}$ Thus, experimental transmittance points versus temperature can be fit with Eq. (1) by leaving $R, d$, and a scale factor as free parameters. Even if the reflection coefficient $R$ is well known in principle (as a function of the refractive index $n$ ), surface imperfections might provoke a small deviation from the theoretical value, thus it is better to leave it as a fitting parameter. A very accurate adjustment of $d$ is also needed, since a small deviation from the value obtained by the interferences method, such as $0.001 \mu \mathrm{m}$, might imply a transmittance change of about $13 \%$.

On the other hand, those dilatational waves which become resonant in the sample introduce variations in the optical path $\Delta(n d)$ leading to oscillations in the transmittance. We can write, then 



FIG. 5. (a) Transmittance versus temperature for a GaSe sample of $20 \mu \mathrm{m}$ thickness. Black triangles are experimental points, the solid line has been obtained by fitting experimental points with formula (1). (b) Derivative of transmittance respect the optical path (solid line, left scale) and experimental oscillation amplitude (black circles, right scale) vs temperature.

$$
\Delta T=\frac{\partial T}{\partial(n d)} \Delta(n d) .
$$

Since $\Delta T$ is proportional to $A_{\text {osc }}$ and should have the same temperature dependence as $\partial T / \partial(n d)$, we can work out the optical path variation introduced by the dilatational waves from Eq. (2). Figure 5(a) shows experimental transmittance points versus temperature and their fitting to Eq. (1), for a sample of $20 \mu \mathrm{m}$ thickness. The solid line in Fig. 5 (b) represents $\partial T / \partial(n d)$. It has been verified that the sign change in the derivative, from positive to negative values, is correlated with a $180^{\circ}$ phase change in the transmittance oscillating signal. Figure 5(b) shows the experimental $A_{\text {osc }}$ values. The evolution of such points with temperature follow Eq. (2) acceptably well even if at $50{ }^{\circ} \mathrm{C}$ the oscillations amplitude increases while the $\partial T / \partial(n d)$ curve tends to zero. In that particular example, the optical path increment $\Delta(n d)$ is about $2.5 \times 10^{-10} \mathrm{~m}$. The same analysis has been performed for different samples and the highest obtained value is $\Delta(n d) \approx 1.4 \times 10^{-9} \mathrm{~m}$.

Different hypothesis have been considered in order to justify the increase in the oscillations amplitude above $50{ }^{\circ} \mathrm{C}$, which has been found in several GaSe samples. A possible cause was initially considered: at higher temperatures the absorption coefficient plays an important role, at $633 \mathrm{~nm}$, and pressure oscillations inside the sample could induce variations of the transmittance through this parameter. Thus another term should be added to Eq. (2): $D$ $=[\partial T / \partial(\alpha d)] \Delta(\alpha d)$. For photons of $1.95 \mathrm{eV}(\lambda=633 \mathrm{~nm})$, $\partial \alpha / \partial P \approx 540 \mathrm{~cm}^{-1} \mathrm{GPa}^{-1}$, and given that $[\partial(n d) / \partial P] / n d$ $\approx-0.0125,{ }^{16}$ at room pressure, we obtain a pressure increment $\Delta P \approx 80 \Delta(n d) / n d$, thus, $\Delta(\alpha d) \approx-2.939$ $\times 10^{4} \Delta(n d)$. This increment multiplied by the derivative $\partial T / \delta(\alpha d)$, calculated from the analytical formula, gives a value for $D$ which is two or three orders of magnitude smaller than $[\partial T / \partial(n d)] \Delta(n d)$, allowing us to conclude that Eq. (2) holds.

A second explanation has been also considered. The probe laser beam may provide an extra power to the acoustic resonance, since the absorption coefficient of GaSe increases sharply at $633 \mathrm{~nm}$, when $t>50{ }^{\circ} \mathrm{C}$. This could give rise to a positive feedback process: a dilatation of the sample combined with an absorbance increase of the probe laser power may, in turn, increase the dilatation. For a sample of $20 \mu \mathrm{m}$, the absorbance at $60{ }^{\circ} \mathrm{C}$ changes from $30 \%$ (minimum) to $50 \%$ (maximum) as a function of the phase. That means a $20 \%$ change for a phase shift of $\pi$ radians. Thus, a $\Delta(n d)$ $\approx 5 \times 10^{-10} \mathrm{~m}$ may produce a $0.06 \%$ absorbance modulation. The probe beam power is $5 \mathrm{~mW}$, hence $3 \mu \mathrm{W}$ of oscillating power would be absorbed in the sample. On the other hand, the pump laser power is $6 \mathrm{~mW}$ in Fig. 5(b). Here we will assume that this power spreads over a frequency interval of about $140 \mathrm{MHz}$, since the pulse width is $7 \mathrm{~ns}$, giving 40 $\mu \mathrm{W} / \mathrm{MHz}$. The previous assumption is obviously a simplification which allows us to elaborate a qualitative prediction. The effective spectral range is determined by other parameters such as the light penetration length or the mechanisms of sound excitation and might be narrower. If we take a 1 $\mathrm{MHz}$ frequency width for the acoustic resonance, then only $40 \mu \mathrm{W}$ of the pump laser would be used to excite the resonance. Moreover, only part of that power is absorbed in the sample since it is smaller than the laser spot and part of the incident power is reflected and transmitted. Thus, we can conclude that our probe beam can be distorting the behavior of GaSe samples at temperatures above $50{ }^{\circ} \mathrm{C}$. In fact, the positive feedback mechanism that we have described has been demonstrated to self-power some acoustic resonances under controlled conditions. ${ }^{17}$

\section{SUMMARY}

In the present work we have used the pump and probe method in order to study the photoacoustic effect in $\mathrm{GaSe}$ and InSe samples. The pump laser pulses generate dilatational waves in the sample, some of which become resonant within its width, leading to periodical variations in the transmittance measured with a probe laser. This resonant phenomenum yields an accurate determination of the longitudinal acoustic-waves velocity parallel to the $c$ axis. The velocity obtained for GaSe and InSe $(2595 \pm 11$ and $2462 \pm 15 \mathrm{~m} / \mathrm{s}$, respectively) is consistent with other values provided by the literature.

\section{ACKNOWLEDGMENTS}

Financial support from DGICYT of Spain (Grants No. TIC93-1203 and No. MAT95-0391) is acknowledged. 
${ }^{1}$ M. V. Andrés, K. W. H. Foulds, and M. J. Tudor, Electron. Lett. 22, 1097 (1986).

${ }^{2}$ A. Rosenewaig, Science 218, 223 (1982).

${ }^{3}$ J. E. Rothenberg, Opt. Lett. 13, 713 (1988).

${ }^{4}$ O. B. Wright, Phys. Rev. B 49, 9985 (1994).

${ }^{5}$ G. S. Kino and R. G. Stearns, Appl. Phys. Lett. 47, 926 (1985).

${ }^{6}$ R. G. Stearns and G. S. Kino, Appl. Phys. Lett. 47, 1048 (1985).

${ }^{7}$ O.B. Wright and V. E. Gusev, Appl. Phys. Lett. 66, 1190 (1995).

${ }^{8}$ C. Thomsen, J. Strait, Z. Vardeny, H. J. Maris, J. Tauc, and J. J. Hauser, Phys. Rev. Lett. 53, 989 (1984).

${ }^{9}$ J. M. Wiesenfeld, Appl. Phys. Lett. 47, 143 (1985)

${ }^{10}$ C. Thomsen, H. T. Grahn, H. J. Maris, and J. Tauc, Phys. Rev. B 34, 4138 (1986).
${ }^{11}$ A. Segura, J. Bouvier, M. V. Andres, and V. Muñoz (unpublished).

${ }^{12}$ B. A. Auld, Acoustic Fields and Waves in Solids, Vol. II (Wiley, New York, 1973), Chap. 10.

${ }^{13}$ Landolt-Börnstein Tables Vol. III/17f, edited by O. Madelung, M. Schulz, and H. Weiss (Springer, Berlin, 1983).

${ }^{14}$ G. I. Abutalybov, S. Z. Dzhafarova, and N. A. Ragimova, Phys. Rev. B 51, 17479 (1995).

${ }^{15}$ M. A. Hernandez, M. V. Andrés, A. Segura, and V. Muñoz, Opt. Commun. 118, 335 (1995)

${ }^{16}$ M. Gauthier, A. Polian, J. M. Besson, and A. Chevy, Phys. Rev. B 40, 3837 (1989).

${ }^{17}$ R. M. Langdon and B. L. Dowe, Proceedings of Fiber Optic Sensor II (The Hague, 1987), pp. 103-52. 